\title{
Pathophysiology of pachyonychia congenita-associated palmoplantar keratoderma: new insights into skin epithelial homeostasis and avenues for treatment*
}

\author{
A.G. Zieman $\mathbb{1}^{1}$ and P.A. Coulombe ${ }^{1,2}$ \\ Departments of ${ }^{1}$ Cell and Developmental Biology and ${ }^{2}$ Dermatology; University of Michigan Medical School, 3071 Biomedical Sciences Research Building, 109 \\ Zina Pitcher Place, Ann Arbor, MI 48109, U.S.A.
}

Linked Comment: Leube and Schwarz. Br J Dermatol 2020; 182:525-526.

\section{Correspondence}

Pierre A. Coulombe.

E-mail: coulombe@umich.edu

Accepted for publication

22 April 2019

\section{Funding sources}

These studies were supported by grant AR044232 issued to P.A.C. from the National Institute of Arthritis, Musculoskeletal and Skin Disease (NIAMS). A.G.Z. received support from grant T32 CA009110 from the National Cancer Institute.

Conflicts of interest

None declared.

*Plain language summary available online

DOI $10.1111 /$ bjd. 18033

\section{Summary}

Background Pachyonychia congenita (PC), a rare genodermatosis, primarily affects ectoderm-derived epithelial appendages and typically includes oral leukokeratosis, nail dystrophy and very painful palmoplantar keratoderma (PPK). PC dramatically impacts quality of life although it does not affect lifespan. PC can arise from mutations in any of the wound-repair-associated keratin genes KRT6A, KRT6B, KRT6C, KRT16 or KRT17. There is no cure for this condition, and current treatment options for PC symptoms are limited and palliative in nature.

Objectives This review focuses on recent progress made towards understanding the pathophysiology of PPK lesions, the most prevalent and debilitating of all PC symptoms.

Methods We reviewed the relevant literature with a particular focus on the Krt16 null mouse, which spontaneously develops footpad lesions that mimic several aspects of PC-associated PPK.

Results There are three main stages of progression of PPK-like lesions in Krt16 null mice. Ahead of lesion onset, keratinocytes in the palmoplantar (footpad) skin exhibit specific defects in terminal differentiation, including loss of Krt9 expression. At the time of PPK onset, there is elevated oxidative stress and hypoactive Keap1-Nrf2 signalling. During active PPK, there is a profound defect in the ability of the epidermis to maintain or return to normal homeostasis.

Conclusions The progress made suggests new avenues to explore for the treatment of PC-based PPK and deepens our understanding of the mechanisms controlling skin tissue homeostasis.

\section{What's already known about this topic?}

- Pachyonychia congenita (PC) is a rare genodermatosis caused by mutations in KRT6A, KRT6B, KRT6C, KRT16 and KRT17, which are normally expressed in skin appendages and induced following injury.

- Individuals with PC present with multiple clinical symptoms that usually include thickened and dystrophic nails, palmoplantar keratoderma (PPK), glandular cysts and oral leukokeratosis.

- The study of PC pathophysiology is made challenging because of its low incidence and high complexity. There is no cure or effective treatment for PC. 


\section{What does this study add?}

- This text reviews recent progress made when studying the pathophysiology of PPK associated with PC.

- This recent progress points to new possibilities for devising effective therapeutics that may complement current palliative strategies.

Pachyonychia congenita (PC; OMIM \#1672000 and 167210) is a rare genodermatosis with a collection of symptoms primarily affecting ectoderm-derived appendages; it includes oral leukokeratosis, nail dystrophies, sebaceous cysts, natal teeth and palmoplantar keratoderma (PPK). While PC does not impact lifespan, it dramatically impacts quality of life for affected individuals. For instance, individuals with PC experience severe plantar pain from PPK lesions daily, often making everyday tasks difficult. There is currently no known cure or effective therapeutics for the treatment of PC. ${ }^{1}$

$\mathrm{PC}$ can arise from autosomal dominant mutations in any of five keratin genes including KRT6A, KRT6B, KRT6C, KRT16 or KRT17. ${ }^{1-5}$ These keratins are normally expressed in epithelial appendages and are otherwise robustly inducible, e.g. after injury or exposure to environmental stresses, together accounting in part for the clinical presentation of this disorder. Most PC-causing mutations are missense alleles, with occasional small insertions or deletions in the keratin coding sequence. Until recently, two major types of PC - type 1 (JadassohnLewandowsky ${ }^{6}$ ) and type 2 (Jackson-Lawler ${ }^{7}$ ) - were recognized based on their prevalent clinical features. Nowadays, five subtypes of PC are recognized based on genetic aetiology - for example, PC caused by a KRT6A mutation corresponds to the PC-K6a subtype. Owing in part to the heterogeneity in the clinical presentation of PC (even among patients with very similar alleles), a definitive diagnosis can be ascertained only through sequencing of these keratin genes. ${ }^{1,8,9}$

\section{The Pachyonychia Congenita Project}

The Pachyonychia Congenita Project is a U.S. public charity that was founded in 2003 and has evolved into a life-changing resource for individuals with PC and for clinicians and researchers interested in this condition. This organization connects individuals with PC and their families to others with this condition, and to clinicians, translational and basic science researchers. The Pachyonychia Congenita Project provides assistance to individuals with PC to attend support meetings and qualify for genetic testing. Further, the Pachyonychia Congenita Project is home to the International Pachyonychia Congenita Research Registry (IPCRR), which gathers extremely valuable data from questionnaires, photos and notes on genetically confirmed PC cases. As of January 2019, the IPCRR includes 864 genetically confirmed cases of PC in 49 countries. This PC registry has evolved into a transformative resource for patients, clinicians and researchers working together towards understanding this disorder and developing effective therapeutics. Finally, the Pachyonychia Congenita Project plays a lead role in fostering basic and clinical research on PC. More information about the Pachyonychia Congenita Project and how to get involved can be found on the publicly available website: www.pachyonychia.org.

\section{Asserting a focus on palmoplantar keratoderma}

While individuals with PC present with many symptoms of significance, PPK is highly penetrant and reportedly the most debilitating (Fig. 1a). ${ }^{1}$ Virtually all individuals with PC $\left(>90 \%{ }^{1}\right)$ present with PPK lesions restricted primarily to pressure points in the palmar and/or plantar epidermis and consisting of dramatic epidermal thickening and hyperkeratosis. ${ }^{3-5,10}$ PPK lesions are debilitating in part because of the extreme pain associated with them. ${ }^{11-13}$ Interestingly, these lesions do not display signs of keratinocyte fragility and/or lysis. The latter represents a predominant element in epidermolysis bullosa simplex (EBS), a genetically determined skin blistering condition caused by mutations in either KRT14 or KRT5. ${ }^{14-17}$ Keratinocyte fragility is also a dominant pathophysiological feature in epidermolytic PPK, which is often caused by mutations in KRT9, ${ }^{18}$ the major differentiation-specific keratin in the volar epidermis. ${ }^{19,20}$ The greater complexity of keratin gene expression in the volar epidermis likely contributes to maintain keratinocyte structural integrity in spite of mutations in individual genes such as KRT6A-C, KRT16 and KRT17. That said, the pathophysiology of PC-associated PPK is only partially understood at present, reflecting significant limitations related to the low incidence of this orphan disease and the severe pain associated with these lesions. ${ }^{21,22}$ Accordingly, there is no effective treatment for PC-based PPK. The current standard of care for PPK consists of routine removal of calluses followed by treatment with moisturizers (see below for details). ${ }^{23}$ A deeper understanding of the pathophysiology of PPK might spearhead the development of effective therapeutics for individuals with PC and also inform researchers, clinicians and drug developers on other genetic and clinical subtypes of PPK (Fig. 1b). This text focuses on recent progress made in deciphering the pathophysiology of PC-associated PPK lesions.

\section{A short primer on the nomenclature of pachyonychia congenita-associated keratin genes}

The original catalogue of human keratin proteins devised by Moll et al. ${ }^{24}$ already recognized the existence of $\mathrm{K} 6$ as a type II 


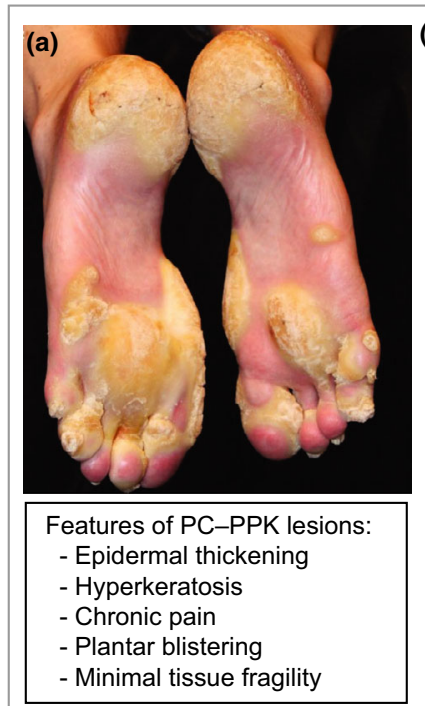

\begin{tabular}{|c|c|c|c|}
\hline (b) Gene family & Genes & PPK subtype & Refs. \\
\hline $\begin{array}{l}\text { Structural } \\
\text { proteins }\end{array}$ & $\begin{array}{l}\text { KRT1, KRT9, } \\
\text { KRT6, KRT14, } \\
\text { KRT16, KRT17 }\end{array}$ & $\begin{array}{l}\text { Pachyonychia congenita, focal nonepidermo- } \\
\text { lytic PPK, epidermolytic PPK, Naegeli-Fran- } \\
\text { ceschetti-Jadassohn syndrome }\end{array}$ & $\begin{array}{l}1-5,18 \\
89-91\end{array}$ \\
\hline $\begin{array}{l}\text { Adhesion } \\
\text { proteins }\end{array}$ & $\begin{array}{l}D S P, P K P 1 \\
D S G 1, J U P\end{array}$ & $\begin{array}{l}\text { McGrath syndrome, keratosis palmoplantaris } \\
\text { striata, Naxos disease }\end{array}$ & $92-96$ \\
\hline $\begin{array}{l}\text { Terminal } \\
\text { differentiation } \\
\text { effectors }\end{array}$ & SERPINB7, LOR & $\begin{array}{l}\text { Nagashima-type PPK, Vohwinkel syndrome, } \\
\text { ichthyosiform variant }\end{array}$ & 97,98 \\
\hline $\begin{array}{l}\text { Ion channels } \\
\& \text { connexins }\end{array}$ & $\begin{array}{l}\text { TRPV3, AQP5, } \\
\text { GJB2, GJA1 }\end{array}$ & $\begin{array}{l}\text { Olmstead syndrome, Bothnian PPK, } \\
\text { oculodentodigital dysplasia, keratitis-ichthyo- } \\
\text { sis-deafness }\end{array}$ & 99-104 \\
\hline Others & $\begin{array}{l}\text { AAGAB, Slurp1, } \\
\text { CARD14, WNT10A, } \\
\text { RHBDF2, CTSC }\end{array}$ & $\begin{array}{l}\text { Type I punctate PPK, Papillon-Fefevre } \\
\text { syndrome, pityriasis pubra pilaris, tylosis with } \\
\text { oesophageal cancer, Schopf-Schulz-Passarge } \\
\text { syndrome, odonto-onycho-dermal dysplasia }\end{array}$ & $\begin{array}{l}105- \\
111\end{array}$ \\
\hline
\end{tabular}

(c)

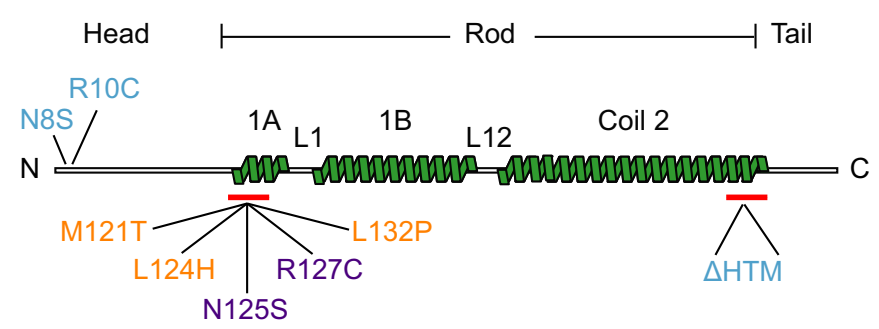

Causative for FNEPPK

Causative for $\mathrm{PC}$

Causative for PC and FNEPPK

Fig 1. PPK, a genetically heterogeneous disorder. (a) Photograph of PC-based PPK lesions from an individual with a KRT16 L124R mutation. Source: Pachyonychia Congenita Project (www.pachyonychia.org). (b) Table summarizing the diversity of genes which, when mutated, can elicit a PPK clinical presentation. Various clinical subtypes of PPK are accounted for. ${ }^{18,89-111}$ (c) Schematic of select mutations in K16 protein that are causative for PC, FNEPPK, or both PC and FNEPPK. K16 exhibits the tripartite domain structure shared by all IF proteins, with an N-terminal 'head' domain, central $\boldsymbol{\alpha}$-helical 'rod' domain and C-terminal 'tail' domain. The central rod domain is comprised of heptad repeat-containing $\boldsymbol{\alpha}$ helical coils (1A, 1B, Coil 2) separated by non-heptad repeat linkers (L1 and L12). Many attributes of the central rod domain (red bars) are highly conserved and represent a signature element among IF proteins. Representative mutations that are causative for FNEPPK are in blue text, mutations causative for PC are in gold text, and mutations that are causative for both FNEPPK and PC are in purple text. FNEPPK, focal nonepidermolytic PPK; IF, intermediate filament; K16, keratin 16; PC, pachyonychia congenita; PPK, palmoplantar keratoderma.

keratin, and of K16 and K17 as type I keratins. However, the true diversity of keratin genes and proteins was underestimated until the advent of whole-genome sequencing efforts, ${ }^{25}$ which necessitated a revision of the Moll nomenclature. ${ }^{26}$ As per the internationally accepted nomenclature, human genes are designated using upper-case lettering (e.g. KRT16) and mouse genes are designated using lower-case lettering (e.g. Krt16). The multiplicity of K6 sequences was originally uncovered in the human. ${ }^{27}$ Currently, we know of two functional genes in the mouse, Krt6a and $\mathrm{Krt} 6 \mathrm{~b},{ }^{28}$ and three functional K6 genes in the human, KRT6A, KRT6B and KRT6C. ${ }^{2,29}$ In contrast, a single gene codes for each of $\mathrm{K} 16^{30,31}$ and $\mathrm{K} 17$ proteins ${ }^{32,33}$ in the human and mouse genomes. The high degree of conservation known to apply to orthologous keratin genes in the mouse and human, in terms of sequence features and regulation, applies to the PC-associated keratin genes. ${ }^{29,31,32}$ This information is relevant to discussing the utilization of transgenic mouse models to study keratin mutation-based human conditions such as PC.

\section{Lessons learned from transgenic mouse models}

As there are no in vitro human cell culture models that can be used to investigate the cellular and molecular mechanisms underlying PPK pathophysiology or screen potential therapeutics, researchers have relied on the use of transgenic mouse models (summarized in Table 1) to study PC and PPK. ${ }^{34}$ Among the models available, the Krt16 null mouse strain is the only one that spontaneously develops footpad skin lesions mimicking PC-associated PPK lesions. Characterization of Krt16 null mice has revealed three phases in PPK, each with a somewhat unique molecular signature: pre-PPK, PPK onset and active PPK (Fig. 2).

In 2-week-old Krt16 null mice, corresponding to the "pre-PPK stage', footpad skin keratinocytes exhibit defects in selective aspects of terminal differentiation. At this early time point there are minimal alterations to the skin tissue histology but, already, a dramatic loss of K9 (Krt9/K9) expression has occurred, which 
Table 1 Mouse models with phenotypes that are potentially relevant to pachyonychia congenita (PC)

\begin{tabular}{|c|c|c|c|c|}
\hline Year & Mouse model & Genetic modification & Main phenotype(s) & References \\
\hline 1996 & Krt6a $\Delta 21 \mathrm{P}$ & $\begin{array}{l}\text { Deletion of } 52 \text { amino acids } \\
\text { (residues 125-176) between } \\
\text { head and 1A helix domain }\end{array}$ & Intraepidermal blistering & 82 \\
\hline 1999 & Krt6a transgenic & $\begin{array}{l}\text { Truncation deleting the } 2 \mathrm{~B} \\
\text { region of the central rod domain }\end{array}$ & Lethal blister or alopecia & 83 \\
\hline 1999 & Krt6a transgenic & Replacement of E2 by HK1-tag & Hyperkeratosis and late-onset alopecia & 83 \\
\hline 2000 & $\mathrm{Krt6a} / \mathrm{Krt}^{-1-}$ & Deletion of Krt6a and Krt6b loci & Oral lesions & 49,84 \\
\hline 2000 & $\mathrm{Krt} \mathrm{a}^{-/-}$ & Deletion of Krt6a loci & $\begin{array}{l}\text { Delay of reepithelialization } \\
\text { after wounding }\end{array}$ & 85 \\
\hline 2002 & $\mathrm{Krt}_{1} 7^{-1-}$ & Deletion of Krt17 locus & Age- and strain-dependent alopecia & 48 \\
\hline 2005 & $\mathrm{Krt6a} / \mathrm{Krt6b}^{-1-} ; \mathrm{Krt}_{1} 7^{-1-}$ & Deletion of Krt6a, Krt6b and Krt17 loci & Severe cell lysis in nail bed epithelium & 86 \\
\hline 2008 & Krt75 knock-in & $\begin{array}{l}\text { Point mutation of codon N158 } \\
\text { (corresponding to mutation } \\
\text { N171 in PC case) }\end{array}$ & Defects in hair shaft, nail fragility & 87 \\
\hline 2011 & KRT6A N171K humanized skin & $\begin{array}{l}\text { Bioengineered skin equivalents derived } \\
\text { from individuals with } \mathrm{PC} \text { with } \mathrm{N} 171 \mathrm{~K} \\
\text { mutation engrafted onto immunodeficient mice }\end{array}$ & Acanthosis and epidermal blistering & 88 \\
\hline 2012 & $\operatorname{Krt} 16^{-1-}$ & Deletion of Krt16 locus & $\begin{array}{l}\text { Oral lesions, footpad lesions } \\
\text { resembling human PPK }\end{array}$ & 38,47 \\
\hline
\end{tabular}

then persists throughout lesion progression. ${ }^{35} \mathrm{Krt9}$ occurs exclusively in differentiating keratinocytes of volar skin and represents a predominant marker gene in this setting. ${ }^{19,20,36}$ In contrast to Krt9, several differentiation markers appear to be upregulated in Krt16 null footpad skin, potentially as a compensatory mechanism. ${ }^{35}$ While this partial defect in terminal differentiation is currently unexplained, ${ }^{35}$ it occurs independent and ahead of the oxidative stress phenotype observed at a later stage of progression of PPK-like lesions in this mouse model. ${ }^{35,37}$

In 1-month-old Krt16 null mice, corresponding to 'onset stage' of PPK-like lesions, footpad skin epidermis displays several features of oxidative stress, including decreased levels of the master cell antioxidant glutathione and decreased expression of glutathione synthesis genes compared with WT controls. Keap1-Nrf2 signalling, a central regulator of the cellular antioxidant response, is markedly attenuated at that time while Nrf2 itself, a transcription factor, is upregulated though ineffective in Krt16 null footpad skin (likely reflecting an attempt to restore redox homeostasis). While difficult to ascertain given restricted access to plantar skin biopsies from patients with PC, there is evidence of reduced Nrf2 activity in PC-PPK lesions of individuals with KRT16 mutations. ${ }^{37}$

In 2-month-old Krt16 null mice, corresponding to an 'active stage' of PPK, there is a profound defect in the ability of footpad skin to maintain or return to normal tissue homeostasis. By this time all Krt16 null mice have spontaneously developed PPK-like lesions on their paws, and while these lesions preferentially arise in areas exposed to the substratum and thus experience mechanical stress, they are not associated with keratinocyte fragility. ${ }^{38,39}$ At a molecular level, the Krt16 null footpad lesions exhibit a gross misregulation of many dangerassociated molecular patterns (DAMPs) and barrier homeostasis genes, which mimics human PC-based PPK lesions. ${ }^{39}$

\section{Lessons learned from computational endeavours}

Along with targeted molecular analyses, computational analysis of genomic datasets has also provided significant insight into the pathophysiology of PC-based PPK. Systems genetics has been used to explore the role of K16 in regulating the skin's response to stress. Re-analysing a powerful systems genetics dataset that related the risk of developing skin tumours to the regulation of skin inflammation and barrier function ${ }^{40}$ revealed a tight link between Krt16, skin barrier genes and innate immunity effectors including DAMPs. ${ }^{39,41}$ Moreover, in this dataset, Krt16 expression is significantly correlated with expression of barrier homeostasis and inflammation genes in tail skin, both at baseline and in response to 12-O-tetradecanoylphorbol-13-acetate (TPA), which acts as a chemical irritant. ${ }^{41}$ The discovery that Krt16 belongs to a network of barrier homeostasis genes pointed to a role for Krt16 in calibrating the skin's response to barrier-compromising stresses, ${ }^{39}$ which converged nicely with the phenotype of PPK-like lesions exhibited by Krt16 null mice. These efforts lent strong support to the notion that a better understanding of how K16 calibrates the skin's stress response could be applicable to PC as KRT16 expression is often elevated in PC-based PPK lesions.

The availability of genome-wide surveys of gene expression from both Krt16 null footpad skin lesions ${ }^{35}$ and PPK lesions from individuals with $\mathrm{PC}^{22}$ has provided an excellent opportunity to further test the strengths and limitations of the Krt16 null mouse as a valid model for PC-based PPK. Merging the human PPK datasets with the murine Krt16 null footpad lesions dataset, based on human-mouse orthologous gene pairings, enabled multiple computational analyses. ${ }^{35}$ Pairwise comparisons of global transcriptional changes in Krt16 null footpad 


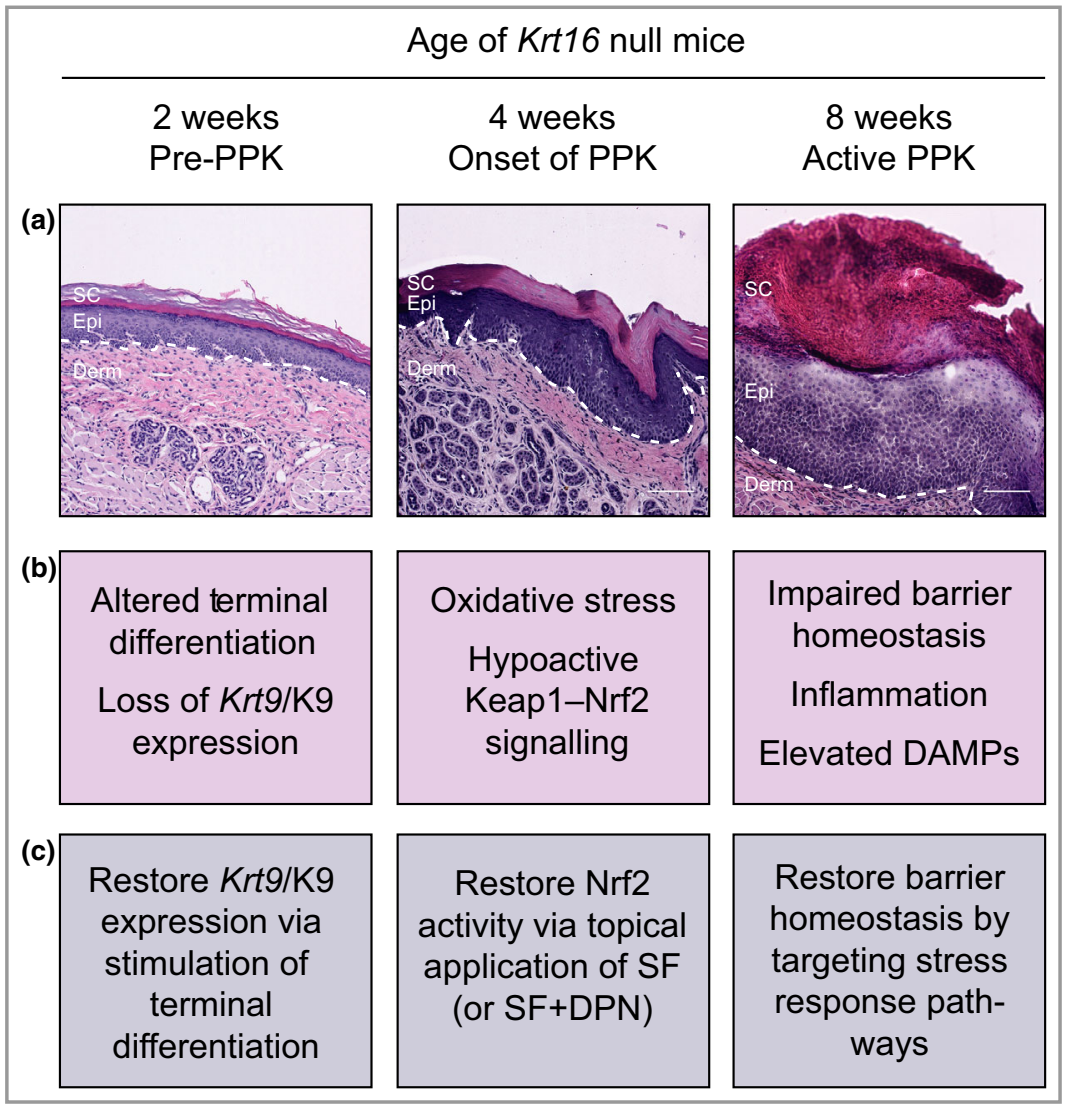

Fig 2. Development of PPK-like lesions in Krt16 null footpad skin proceeds in three stages: pre-PPK (at 2 weeks of age), onset of PPK (at 4 weeks) and active PPK (at 8 weeks). (a) Representative histology of Krt16 null footpad skin at 2, 4 and 8 weeks of age. At 2 weeks, the epidermis shows a normal thickness and overall architecture but, on closer inspection, alterations including the abnormal appearance of the granular layer, crowding of basal keratinocytes, and a decreased nuclear aspect ratio of basal keratinocytes can be seen. At 4 weeks, prior to macroscopic appearance of lesions, mild epidermal thickening is observed. By 8 weeks, there is dramatic thickening of the living epidermis (Epi) and the stratum corneum (SC), infiltration of immune cells, and limited suprabasal cell lysis. The dotted line shows the epidermal-dermal junction. Scale bar $=100 \mu \mathrm{m}$. Images acquired using a Zeiss microscope with Apotome attachment and processed using Zen 2.3 software. (b) Summary of key molecular changes that occur at 2, 4 and 8 weeks of age in Krt16 null footpad skin. ${ }^{35,37-39,47,77}$ (c) Potential therapeutic interventions for each stage of lesion development. ${ }^{35,37,39,77}$ DAMPs, damage-associated molecular pattern molecules; Derm, dermis; DPN, diarylpropionitrile; PPK, palmoplantar keratoderma; SF, sulforaphane.

lesions and individual PC cases (three KRT6 cases, three KRT16 cases) generated statistically significant positive correlation values in all cases (Fig. 3a,b). Additionally, pairwise comparisons of global transcriptional changes further highlight the high degree of heterogeneity between individual cases involving different keratin mutations, and between cases with the same mutated keratin allele (Fig. 3c). Altogether these comparisons provided a strong case that lesional Krt16 null mouse footpad skin mimics PC-associated PPK lesions at a global gene expression level, reinforcing and extending the notion that the Krt16 null mouse is an appropriate model for the study of pathogenesis of PC-associated PPK lesions.

\section{A role for keratin imbalances and genetic background in the pathophysiology of palmoplantar keratoderma}

Because the presentation of PC symptoms varies greatly between individuals even with similar or the same mutated keratin allele, ${ }^{1,42-44}$ there is likely a role for genetic background and gene modifiers in the pathophysiology of this condition. Remarkably, similar alleles in KRT16 (N125S and R127C) can elicit a presentation of focal nonepidermolytic PPK vs. full-blown PC (Fig. 1c), ${ }^{44-46}$ suggesting that the consequences associated with alterations in KRT16 are subject to modifier gene(s) effects. Consistent with this notion, despite the immunological differences between mice and humans, several phenotypic aspects of Krt16 null mice including the PPK-like lesions are modestly impacted by genetic strain background. ${ }^{47}$ Interestingly, select phenotypic traits in Krt17 null mice $^{48}$ and Krt6a/Krt6b double-null mice ${ }^{49}$ also exhibit a dependence on genetic background.

In addition to genetic background, imbalances in keratin expression also appear likely to play a significant role in the pathophysiology of PC-based PPK. For example, the differentiation-specific keratins KRT2 and KRT9 are both decreased in Krt16 null footpad lesions and human PC-based PPK. ${ }^{22,35,50}$ Of note, mice that are double-null for the differentiation-specific 


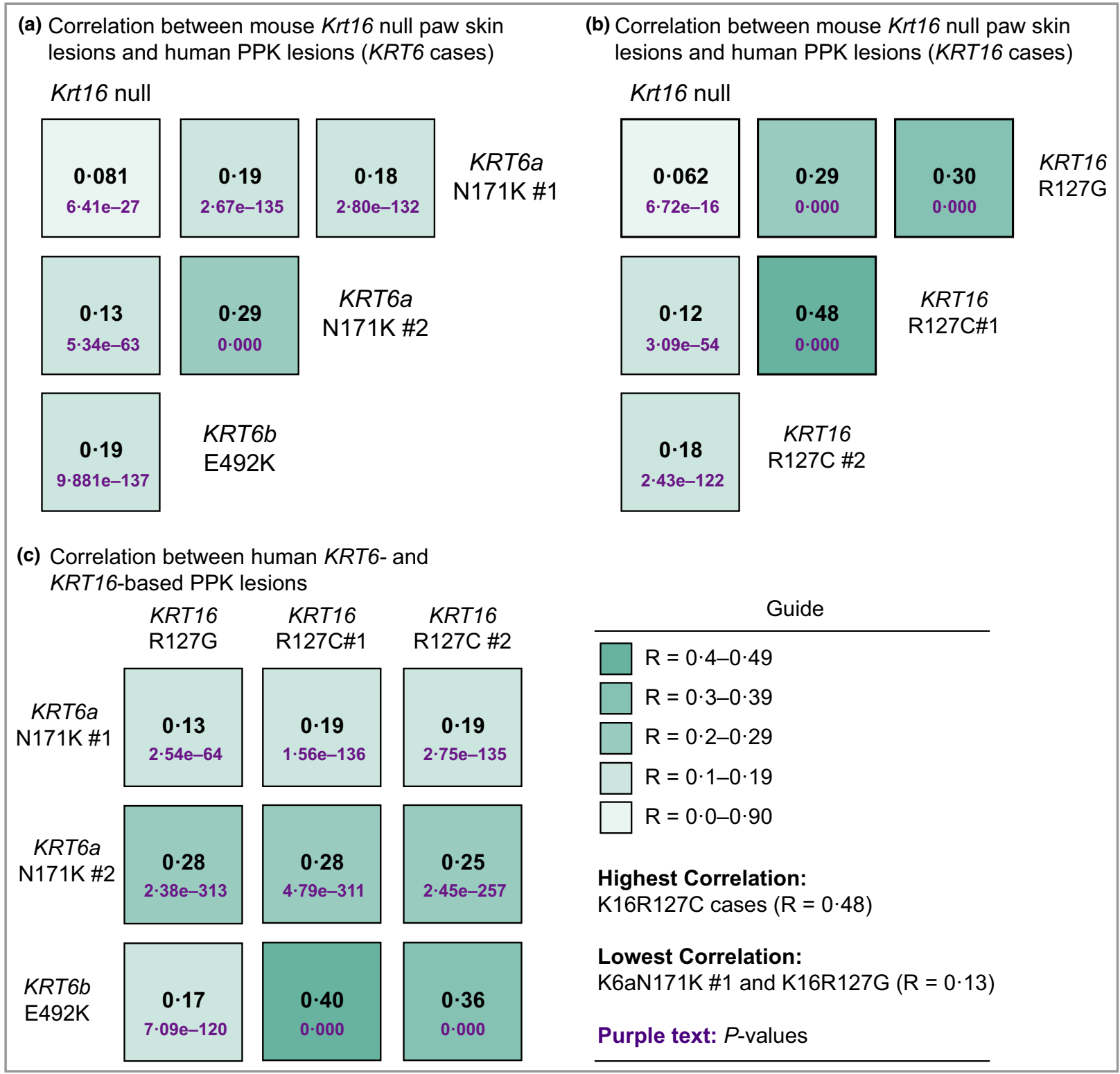

Fig 3. Correlation of transcriptional changes between Krt16 null footpad lesions and human PC-based PPK lesions. (a,b) Correlation coefficients (R) calculated from pairwise comparisons of microarray data from Krt16 null footpad lesions ${ }^{35}$ and human PC-based PPK lesions ${ }^{22}$ resulting from mutations in KRT6 (a) or KRT16 (b). (c) Correlation coefficients calculated from pairwise comparisons between individual cases of PC-based PPK with either KRT6 or KRT16 mutations. An R-value of -1 would convey perfect negative correlation, whereas an R-value of 1 conveys perfect correlation between samples. P-values for each correlation are denoted in purple text underneath the corresponding R-values. Figure adapted from Zieman et al. ${ }^{35}$ PC, pachyonychia congenita; PPK, palmoplantar keratoderma

Krt2 and Krt10 develop a keratoderma-like phenotype on footpad $\operatorname{skin}^{51}$ while mice null for Krt9 develop an epidermolytic PPK that closely resembles the corresponding human disorder. $^{36}$ Aside from these (and other) alterations, ${ }^{22,35}$ the expression of KRT6A, KRT6B, KRT16 and/or KRT17 (including the mutated alleles) is dramatically increased in PC-based PPK, as expected given the stress- and wound-sensitive regulation of these genes. ${ }^{22}$ Given the knowledge that K6, K16 and K17 proteins have pleiotropic and context-dependent properties, ${ }^{35,37,39,52-56}$ such alterations in keratin protein levels and balance among them are poised to have a striking impact on the development and evolution of PPK lesions.

\section{Pathophysiological unknowns in palmoplantar keratoderma and other clinical features of pachyonychia congenita}

While all clinical manifestations associated with PC are worth a deep investigation, two stand out as remaining particularly intriguing at a cellular and molecular level. One is 
the occurrence of individual or multiple cysts (steatocystoma multiplex; see OMIM entries \#184500 and \#184510) in patients with PC, which are benign fluid-filled cysts believed to originate from sebaceous glands and which can occur all over the body and arise preferentially in individuals with mutations in KRT17. ${ }^{57-59}$ These cysts often require surgical drainage or removal as their rupture and/or inflammation pose a risk of infection and can be painful for patients. ${ }^{1}$ Another intriguing manifestation is natal teeth, which refers to presence of teeth in newborns and is also preferentially associated with mutations in KRT17. ${ }^{1,59}$ Natal teeth are soft, friable and prone to caries, and are usually lost within the first few months of life. ${ }^{1,60-63}$ Of note, Krt17 is expressed at a very early stage of the development of ectodermal appendages, including the tooth. ${ }^{32}$ Recent studies have shown that genetic variants in the PC-associated keratin genes increase susceptibility to tooth decay. ${ }^{64}$ There is currently no model to study the cystic skin lesions and phenomenon of natal teeth associated with PC.

\section{Limitations of past and current therapeutic strategies for pachyonychia congenita}

PC-associated PPK has been treated with a combination of keratolytics, pain medication, orthotics and mechanical removal of calluses. ${ }^{23,65}$ While the keratolytics salicylic acid and urea soften calluses, they cannot control the significant overgrowth associated with most cases of PC-PPK. Pain medication and custom orthotics can partially alleviate discomfort, but do not treat the underlying PPK. Routine mechanical removal of calluses by filing, grinding or cutting has been the most satisfying treatment for individuals with $\mathrm{PC} .^{23}$ Significant efforts are currently under way to develop new and effective therapeutics for the management of these lesions. Two distinct strategies are highlighted here. The first strategy involves the development of short interfering RNAs (siRNAs) that specifically target mutant keratin alleles and reduce their expression. It has shown some promise in a trial of the siRNA TD101, which targets the KRT6A N171K allele, albeit in a single patient. ${ }^{21,66,67}$ In its current form, this approach suffers from the limitation that delivery of such nucleic acid-based therapeutics requires intradermal injections that cause intense pain to the patient. The generation of self-delivery siRNAs for mutant keratin alleles improves the uptake of siRNAs by keratinocytes $^{68}$ but does not improve penetration through the stratum corneum. Accordingly, a method to deliver siRNAbased therapeutics that involves topical application of therapeutic agents is sorely needed. ${ }^{69}$

The second strategy to treat PC-based PPK consists of drugbased interventions aimed at reducing mutant keratin gene expression. The mammalian target of rapamycin (mTOR) inhibitor rapamycin/sirolimus suppresses K6a expression and, when taken orally, improves PC symptoms. ${ }^{70}$ However, severe side-effects associated with systemic rapamycin treatment prevent it from being a viable long-term treatment for PC. Recently, topical sirolimus treatment of two patients with
K6a-based disease improved PC-PPK without the toxicity of systemic treatment ${ }^{71}$ but requires additional studies to confirm the safety and efficacy of this treatment. Oral retinoids successfully reduced callus thickness in some individuals with PC, but like rapamycin, adverse side-effects including increased pain prevent oral retinoids from being a viable long-term treatment for PC. ${ }^{72}$ Statins can also downregulate KRT6A expression, ${ }^{73}$ but so far only oral rosuvastatin has been shown to be effective in a single case of K6a-based PC. ${ }^{74}$ Finally, injections of botulinum toxin (Botox) into plantar calluses improved plantar blistering and pain associated with PC-PPK lesions, ${ }^{75}$ but injections are costly and must be performed under anaesthesia. While each of these drug-based interventions provides some relief, none of them in present form provide viable long-term treatment strategies for PC-based PPK.

\section{Opportunities for novel therapies}

A promising opportunity to complement ongoing efforts to develop effective therapeutics for PC-based PPK would be to target stress response pathways and/or pathways capable of promoting the restoration of normal epidermal differentiation. In Krt16 null mice, topical treatment with the small natural molecule sulforaphane (SF), which activates Nrf2 signalling by modifying Keap1, ${ }^{76}$ can prevent PPK-like lesions in male mice. ${ }^{37}$ Addition of the ER- $\beta$ agonist diarylpropionitrile to the $\mathrm{SF}$ treatment regimen is necessary for successful activation of $\mathrm{Nrf} 2^{77}$ and prevention of PPK-like lesions in female mice. SF is available in pure form or as part of broccoli sprout extract, ${ }^{78}$ can be safely delivered topically, and has shown therapeutic promise in the treatment of EBS arising from mutations in either keratins $\mathrm{K} 5$ or $\mathrm{K} 14 .^{79,80}$ The sexual dimorphism in response to SF treatment in mice is a reminder that sex-based differences are important considerations when developing therapeutics for any disease. ${ }^{81}$ Whether there is a sexual dimorphism in the setting of PC remains an open question.

Another strategy worth considering is to normalize terminal differentiation in volar skin. In male Krt16 null mice treated with SF prior to lesion onset, restoration of Nrf2 activity coincided with induction of Krt9 expression. ${ }^{35}$ Additional efforts should be focused on testing this specific strategy. In the end, the prospect of combining treatment modalities that act to prevent and/or treat active lesions represent an attractive prospect for the treatment of a condition featuring the complexity of PC-associated PPK.

\section{Future directions}

$\mathrm{PC}$ is a monogenic skin disease with a complex, polygenic presentation. Despite the plethora of challenges that arise in studying this disease, the use of transgenic mouse models and of computational biology has been invaluable and has provided novel insight into the pathophysiology of PC-based PPK, one of the most debilitating symptoms for individuals with PC. The study of PPK pathophysiology not only paves the way for researchers to devise therapeutics to treat $\mathrm{PC}$, but also 
provides an opportunity to better understand the mechanisms that control skin tissue homeostasis.

\section{Acknowledgments}

The authors wish to thank members of the Coulombe laboratory for their support. This work was supported by grant AR044232 from the National Institutes of Health.

\section{References}

1 Leachman SA, Kaspar RL, Fleckman P et al. Clinical and pathological features of pachyonychia congenita. J Invest Dermatol 2005; 10:3-17.

2 Wilson NJ, Messenger AG, Leachman SA et al. Keratin K6c mutations cause focal palmoplantar keratoderma. J Invest Dermatol 2010; 130:425-9.

3 McLean WH, Rugg EL, Lunny DP et al. Keratin 16 and keratin 17 mutations cause pachyonychia congenita. Nat Genet 1995; 9:273-8.

4 Smith FJ, Jonkman MF, van Goor $\mathrm{H}$ et al. A mutation in human keratin K6b produces a phenocopy of the K17 disorder pachyonychia congenita type 2. Hum Mol Genet 1998; 7:1143-8.

5 Bowden PE, Haley JL, Kansky A et al. Mutation of a type II keratin gene (K6a) in pachyonychia congenita. Nat Genet 1995; 10:363-5.

6 Franklin J. Pachyonychia congenita (Jadassohn and Lewandowski). Proc R Soc Med 1939; 32:263-5.

7 Jackson AD, Lawler SD. Pachyonychia congenita; a report of six cases in one family, with a note on linkage data. Ann Eugen 1951; 16:142-6.

8 Liao H, Sayers JM, Wilson NJ et al. A spectrum of mutations in keratins K6a, K16 and K17 causing pachyonychia congenita. J Dermatol Sci 2007; 48:199-205.

9 Fu T, Leachman SA, Wilson NJ et al. Genotype-phenotype correlations among pachyonychia congenita patients with K16 mutations. J Invest Dermatol 2011; 131:1025-8.

10 Lin MT, Levy ML, Bowden PE et al. Identification of sporadic mutations in the helix initiation motif of keratin 6 in two pachyonychia congenita patients: further evidence for a mutational hot spot. Exp Dermatol 1999; 8:115-19.

11 Krupiczojc MA, O’Toole EA. Plantar pain in pachyonychia congenita. Br J Dermatol 2018; 179:11-12.

12 Brill S, Sprecher E, Smith FJD et al. Chronic pain in pachyonychia congenita: evidence for neuropathic origin. Br J Dermatol 2018; 179:154-62.

13 Weinberg RL, Coulombe PA, Polydefkis M, Caterina MJ. Pain mechanisms in hereditary palmoplantar keratodermas. Br J Dermatol 2020; 182:543-51.

14 Coulombe PA, Fuchs E. Epidermolysis bullosa simplex. Sem in Dermatol 1993; 12:173-90.

15 Coulombe PA, Hutton ME, Letai A et al. Point mutations in human keratin 14 genes of epidermolysis bullosa simplex patients: genetic and functional analyses. Cell 1991; 66:1301-11.

16 Coulombe PA, Hutton ME, Vassar R et al. A function for keratins and a common thread among different types of epidermolysis bullosa simplex diseases. J Cell Biol 1991; 115:1661-74.

17 Coulombe PA, Kerns ML, Fuchs E. Epidermolysis bullosa simplex: a paradigm for disorders of tissue fragility. J Clin Invest 2009; 119:1784-93.

18 Reis A, Hennies HC, Langbein L et al. Keratin 9 gene mutations in epidermolytic palmoplantar keratoderma (EPPK). Nat Genet 1994; 6:174-9.
19 Langbein L, Heid HW, Moll I, Franke WW. Molecular characterization of the body site-specific human epidermal cytokeratin 9: cDNA cloning, amino acid sequence, and tissue specificity of gene expression. Differentiation 1993; 55:57-71.

20 Kim D, Hossain MZ, Nieves A et al. To control site-specific skin gene expression, autocrine mimics paracrine canonical Wnt signaling and is activated ectopically in skin disease. Am J Pathol 2016; 186:1140-50.

21 Leachman SA, Hickerson RP, Schwartz ME et al. First-in-human mutation-targeted siRNA phase $\mathrm{Ib}$ trial of an inherited skin disorder. Mol Ther 2010; 18:442-6.

22 Cao YA, Hickerson RP, Seegmiller BL et al. Gene expression profiling in pachyonychia congenita skin. J Dermatol Sci 2015; 77:15665 .

23 Goldberg I, Fruchter D, Meilick A et al. Best treatment practices for pachyonychia congenita. J Eur Acad Dermatol Venereol 2014; 28:279-85.

24 Moll R, Franke WW, Schiller DL et al. The catalog of human cytokeratins: patterns of expression in normal epithelia, tumors and cultured cells. Cell 1982; 31:11-24.

25 Hesse M, Magin TM, Weber K. Genes for intermediate filament proteins and the draft sequence of the human genome: novel keratin genes and a surprisingly high number of pseudogenes related to keratin genes 8 and 18. J Cell Sci 2001; 114:2569-75.

26 Schweizer J, Bowden PE, Coulombe PA et al. New consensus nomenclature for mammalian keratins. J Cell Biol 2006; 174:16974.

27 Tyner AL, Fuchs E. Evidence for posttranscriptional regulation of the keratins expressed during hyperproliferation and malignant transformation in human epidermis. J Cell Biol 1986; 103:194555.

28 Takahashi K, Yan B, Yamanishi K et al. The two functional keratin 6 genes of mouse are differentially regulated and evolved independently from their human orthologs. Genomics 1998; 53:17083.

29 Takahashi K, Paladini RD, Coulombe PA. Cloning and characterization of multiple human genes and cDNAs encoding highly related type II keratin 6 isoforms. J Biol Chem 1995; 270:1858192.

30 Rosenberg M, RayChaudhury A, Shows TB et al. A group of type I keratin genes on human chromosome 17: characterization and expression. Mol Cell Biol 1988; 8:722-36.

31 Bernot KM, Coulombe PA, McGowan KM. Keratin 16 expression defines a subset of epithelial cells during skin morphogenesis and the hair cycle. J Investig Dermatol 2002; 119:1137-49.

32 McGowan KM, Coulombe PA. Onset of keratin 17 expression coincides with the definition of major epithelial lineages during skin development. J Cell Biol 1998; 143:469-86.

33 Troyanovsky SM, Leube RE, Franke WW. Characterization of the human gene encoding cytokeratin 17 and its expression pattern. Eur J Cell Biol 1992; 59:127-37.

34 Chen J, Roop DR. Mouse models in preclinical studies for pachyonychia congenita. J Investig Dermatol Symp Proc 2005; 10:37-46.

35 Zieman AG, Poll BG, Ma J, Coulombe PA. Altered keratinocyte differentiation is an early driver of keratin mutation-based palmoplantar keratoderma. Hum Mol Genet 2019; 28:2255-70.

$36 \mathrm{Fu}$ DJ, Thomson C, Lunny DP et al. Keratin 9 is required for the structural integrity and terminal differentiation of the palmoplantar epidermis. J Investig Dermatol 2014; 134:754-63.

37 Kerns ML, Hakim JM, Lu RG et al. Oxidative stress and dysfunctional NRF2 underlie pachyonychia congenita phenotypes. J Clin Invest 2016; 126:2356-66. 
38 Lessard JC, Coulombe PA. Keratin 16-null mice develop palmoplantar keratoderma, a hallmark feature of pachyonychia congenita and related disorders. J Investig Dermatol 2012; 132:1384-91.

39 Lessard JC, Pina-Paz S, Rotty JD et al. Keratin 16 regulates innate immunity in response to epidermal barrier breach. Proc Natl Acad Sci U S A 2013; 110:19537-42.

40 Quigley DA, To MD, Perez-Losada J et al. Genetic architecture of mouse skin inflammation and tumour susceptibility. Nature 2009; 458:505-8.

41 Quigley DA, Kandyba E, Huang P et al. Gene expression architecture of mouse dorsal and tail skin reveals functional differences in inflammation and cancer. Cell Reports 2016; 16:1153-65.

42 Covello SP, Smith FJ, Sillevis Smitt JH et al. Keratin 17 mutations cause either steatocystoma multiplex or pachyonychia congenita type 2. Br J Dermatol 1998; 139:475-80.

43 Smith FJ, Corden LD, Rugg EL et al. Missense mutations in keratin 17 cause either pachyonychia congenita type 2 or a phenotype resembling steatocystoma multiplex. J Investig Dermatol 1997; 108:220-3.

44 Smith FJ, Fisher MP, Healy E et al. Novel keratin 16 mutations and protein expression studies in pachyonychia congenita type 1 and focal palmoplantar keratoderma. Exp Dermatol 2000; 9:170-7.

45 Shamsher MK, Navsaria HA, Stevens HP et al. Novel mutations in keratin 16 gene underly focal non-epidermolytic palmoplantar keratoderma (NEPPK) in two families. Hum Mol Genet 1995; 4:1875-81.

46 Smith FJ, Liao H, Cassidy AJ et al. The genetic basis of pachyonychia congenita. J Investig Dermatol Symp Proc 2005; 10:21-30.

47 Zieman A, Coulombe PA. The keratin 16 null phenotype is modestly impacted by genetic strain background in mice. Exp Dermatol 2018; 27:672-4.

48 McGowan KM, Tong X, Colucci-Guyon E et al. Keratin 17 null mice exhibit age- and strain-dependent alopecia. Genes Dev 2002; 16:1412-22.

49 Wong P, Colucci-Guyon E, Takahashi $\mathrm{K}$ et al. Introducing a null mutation in the mouse K6alpha and K6beta genes reveals their essential structural role in the oral mucosa. J Cell Biol 2000; 150:921-8.

50 Rice RH, Durbin-Johnson BP, Salemi M et al. Proteomic profiling of pachyonychia congenita plantar callus. J Proteomics 2017; 165: 132-7.

51 Fischer H, Langbein L, Reichelt J et al. Keratins K2 and K10 are essential for the epidermal integrity of plantar skin. J Dermatol Sci 2016; 81:10-16.

52 Tong X, Coulombe PA. Keratin 17 modulates hair follicle cycling in a TNFalpha-dependent fashion. Genes Dev 2006; 20:1353-64.

53 Depianto D, Kerns ML, Dlugosz AA et al. Keratin 17 promotes epithelial proliferation and tumor growth by polarizing the immune response in skin. Nat Gen 2010; 42:910-14.

54 Rotty JD, Coulombe PA. A wound-induced keratin inhibits Src activity during keratinocyte migration and tissue repair. J Cell Biol 2012; 197:381-9.

55 Chung BM, Arutyunov A, Ilagan E et al. Regulation of C-X-C chemokine gene expression by keratin 17 and hnRNP $\mathrm{K}$ in skin tumor keratinocytes. J Cell Biol 2015; 208:613-27.

56 Hobbs RP, DePianto DJ, Jacob JT et al. Keratin-dependent regulation of Aire and gene expression in skin tumor keratinocytes. Nat Gen 2015; 47:933-8.

57 McLean WH, Hansen CD, Eliason MJ et al. The phenotypic and molecular genetic features of pachyonychia congenita. J Investig Dermatol 2011; 131:1015-17.

58 Wilson NJ, Leachman SA, Hansen CD et al. A large mutational study in pachyonychia congenita. J Investig Dermatol 2011; 131:1018-24.
59 Eliason MJ, Leachman SA, Feng BJ et al. A review of the clinical phenotype of 254 patients with genetically confirmed pachyonychia congenita. J Am Acad Dermatol 2012; 67:680-6.

60 Clementi M, Cardin de Stefani E, Dei Rossi C et al. Pachyonychia congenita Jackson-Lawler type: a distinct malformation syndrome. Br J Dermatol 1986; 114:367-70.

61 Feinstein A, Friedman J, Schewach-Millet M. Pachyonychia congenita. J Am Acad Dermatol 1988; 19:705-11.

62 Munro CS, Carter S, Bryce $S$ et al. A gene for pachyonychia congenita is closely linked to the keratin gene cluster on $17 \mathrm{q} 12-\mathrm{q} 21$. J Med Genet 1994; 31:675-8.

63 Terrinoni A, Smith FJ, Didona B et al. Novel and recurrent mutations in the genes encoding keratins K6a, K16 and K17 in 13 cases of pachyonychia congenita. J Investig Dermatol 2001; 117:1391-6.

64 Duverger O, Carlson JC, Karacz CM et al. Genetic variants in pachyonychia congenita-associated keratins increase susceptibility to tooth decay. PLoS Genet 2018; 14:e1007168.

65 Porter RM, Bravo AA, Smith FJD. Management of plantar keratodermas: lessons from pachyonychia congenita. J Am Podiatr Med Assoc 2017; 107:428-35.

66 Leachman SA, Hickerson RP, Hull PR et al. Therapeutic siRNAs for dominant genetic skin disorders including pachyonychia congenita. J Dermatol Sci 2008; 51:151-7.

67 Hickerson RP, Smith FJ, Reeves RE et al. Single-nucleotide-specific siRNA targeting in a dominant-negative skin model. J Investig Dermatol 2008; 128:594-605.

68 Hickerson RP, Flores MA, Leake D et al. Use of self-delivery siRNAs to inhibit gene expression in an organotypic pachyonychia congenita model. J Investig Dermatol 2011; 131:1037-44.

69 Kaspar RL, Leachman SA, McLean WH et al. Toward a treatment for pachyonychia congenita: report on the 7th Annual International Pachyonychia Congenita Consortium meeting. J Investig Dermatol 2011; 131:1011-14.

70 Hickerson RP, Leake D, Pho LN et al. Rapamycin selectively inhibits expression of an inducible keratin (K6a) in human keratinocytes and improves symptoms in pachyonychia congenita patients. J Dermatol Sci 2009; 56:82-8.

71 Teng JMC, Bartholomew FB, Patel V et al. Novel treatment of painful plantar keratoderma in pachyonychia congenita using topical sirolimus. Clin Exp Dermatol 2018; 43:968-71.

72 Gruber R, Edlinger M, Kaspar RL et al. An appraisal of oral retinoids in the treatment of pachyonychia congenita. J Am Acad Dermatol 2012; 66:e193-9.

73 Zhao Y, Gartner U, Smith FJ et al. Statins downregulate K6a promoter activity: a possible therapeutic avenue for pachyonychia congenita. J Investig Dermatol 2011; 131:1045-52.

74 Abdollahimajd F, Rajabi F, Shahidi-Dadras M et al. Pachyonychia congenita: a case report of a successful treatment with rosuvastatin in a patient with a KRT6A mutation. Br J Dermatol 2019; 181:584-6.

75 Swartling C, Karlqvist M, Hymnelius $\mathrm{K}$ et al. Botulinum toxin in the treatment of sweat-worsened foot problems in patients with epidermolysis bullosa simplex and pachyonychia congenita. Br J Dermatol 2010; 163:1072-6.

$76 \mathrm{Hu}$ C, Eggler AL, Mesecar AD, van Breemen RB. Modification of keap1 cysteine residues by sulforaphane. Chem Res Toxicol 2011; 24:515-21.

77 Kerns ML, Hakim JMC, Zieman A et al. Sexual dimorphism in response to an NRF2 inducer in a model for pachyonychia congenita. J Investig Dermatol 2018; 138:1094-100.

78 Zhang Y, Talalay P, Cho CG et al. A major inducer of anticarcinogenic protective enzymes from broccoli: isolation and elucidation of structure. Proc Natl Acad Sci U S A 1992; 89:2399-403. 
79 Kerns ML, DePianto D, Dinkova-Kostova AT et al. Reprogramming of keratin biosynthesis by sulforaphane restores skin integrity in epidermolysis bullosa simplex. Proc Natl Acad Sci U S A 2007; 104: 14460-5.

80 Kerns ML, Guss L, Fahey J et al. Randomized, split-body, singleblinded clinical trial of topical broccoli sprout extract: assessing the feasibility of its use in keratin-based disorders. J Am Acad Dermatol 2017; 76:449-53.e1.

81 Leube RE, Schwarz N. Sex matters: interfering with the oxidative stress response in pachyonychia congenita. J Investig Dermatol 2018; 138: 1019-22.

82 Takahashi K, Coulombe PA. A transgenic mouse model with an inducible skin blistering disease phenotype. Proc Natl Acad Sci U S A 1996; 93:14776-81.

83 Wojcik SM, Imakado S, Seki T et al. Expression of MK6a dominant-negative and C-terminal mutant transgenes in mice has distinct phenotypic consequences in the epidermis and hair follicle. Differentiation 1999; 65:97-112.

84 Wojcik SM, Longley MA, Roop DR. Discovery of a novel murine keratin 6 (K6) isoform explains the absence of hair and nail defects in mice deficient for K6a and K6b. J Cell Biol 2001; 154:619-30.

85 Wojcik SM, Bundman DS, Roop DR. Delayed wound healing in keratin 6a knockout mice. Mol Cell Biol 2000; 20:5248-55.

86 Wong $\mathrm{P}$, Domergue R, Coulombe PA. Overcoming functional redundancy to elicit pachyonychia congenita-like nail lesions in transgenic mice. Mol Cell Biol 2005; 25:197-205.

87 Chen J, Jaeger K, Den Z et al. Mice expressing a mutant Krt75 (K6hf) allele develop hair and nail defects resembling pachyonychia congenita. J Investig Dermatol 2008; 128:270-9.

88 Garcia M, Larcher F, Hickerson RP et al. Development of skinhumanized mouse models of pachyonychia congenita. J Investig Dermatol 2011; 131:1053-60.

89 Kimonis V, DiGiovanna JJ, Yang JM et al. A mutation in the V1 end domain of keratin 1 in non-epidermolytic palmar-plantar keratoderma. J Investig Dermatol 1994; 103:764-9.

90 Arin MJ, Longley MA, Kuster W et al. An asparagine to threonine substitution in the $1 \mathrm{~A}$ domain of keratin 1: a novel mutation that causes epidermolytic hyperkeratosis. Exp Dermatol 1999; 8:124-7.

91 Lugassy J, Itin P, Ishida-Yamamoto A et al. Naegeli-FranceschettiJadassohn syndrome and dermatopathia pigmentosa reticularis: two allelic ectodermal dysplasias caused by dominant mutations in KRT14. Am J Hum Genet 2006; 79:724-30.

92 Armstrong DK, McKenna KE, Purkis PE et al. Haploinsufficiency of desmoplakin causes a striate subtype of palmoplantar keratoderma. Hum Mol Genet 1999; 8:143-8.

93 Rickman L, Simrak D, Stevens HP et al. N-terminal deletion in a desmosomal cadherin causes the autosomal dominant skin disease striate palmoplantar keratoderma. Hum Mol Genet 1999; 8:971-6.

94 McGrath JA, McMillan JR, Shemanko CS et al. Mutations in the plakophilin 1 gene result in ectodermal dysplasia/skin fragility syndrome. Nat Genet 1997; 17:240-4.

95 Whittock NV, Smith FJ, Wan H et al. Frameshift mutation in the V2 domain of human keratin 1 results in striate palmoplantar keratoderma. J Investig Dermatol 2002; 118:838-44.

96 McKoy G, Protonotarios N, Crosby A et al. Identification of a deletion in plakoglobin in arrhythmogenic right ventricular cardiomyopathy with palmoplantar keratoderma and woolly hair (Naxos disease). Lancet 2000; 355:2119-24.
97 Kubo A, Shiohama A, Sasaki $\mathrm{T}$ et al. Mutations in SERPINB7, encoding a member of the serine protease inhibitor superfamily, cause Nagashima-type palmoplantar keratosis. Am J Hum Genet 2013; 93:945-56.

98 Maestrini E, Monaco AP, McGrath JA et al. A molecular defect in loricrin, the major component of the cornified cell envelope, underlies Vohwinkel's syndrome. Nat Genet 1996; 13:70-7.

99 Lin Z, Chen Q, Lee $M$ et al. Exome sequencing reveals mutations in TRPV3 as a cause of Olmsted syndrome. Am J Hum Genet 2012; 90:558-64.

100 Lind L, Lundstrom A, Hofer PA et al. The gene for diffuse palmoplantar keratoderma of the type found in northern Sweden is localized to chromosome 12q11-q13. Hum Mol Genet 1994; 3:1789-93.

101 Blaydon DC, Lind LK, Plagnol V et al. Mutations in AQP5, encoding a water-channel protein, cause autosomal-dominant diffuse nonepidermolytic palmoplantar keratoderma. Am J Hum Genet 2013; 93:330-5.

102 Richard G, White TW, Smith LE et al. Functional defects of Cx26 resulting from a heterozygous missense mutation in a family with dominant deaf-mutism and palmoplantar keratoderma. Hum Genet 1998; 103:393-9.

103 van Steensel MA, Spruijt L, van der Burgt I et al. A 2-bp deletion in the GJA1 gene is associated with oculo-dento-digital dysplasia with palmoplantar keratoderma. Am J Med Genet A 2005; 132A: $171-4$.

104 Gong XQ, Shao Q, Lounsbury CS et al. Functional characterization of a GJA1 frameshift mutation causing oculodentodigital dysplasia and palmoplantar keratoderma. J Biol Chem 2006; 281:31801-11.

105 Giehl KA, Eckstein GN, Pasternack SM et al. Nonsense mutations in $A A G A B$ cause punctate palmoplantar keratoderma type Buschke-Fischer-Brauer. Am J Hum Genet 2012; 91:754-9.

106 Fischer J, Bouadjar B, Heilig R et al. Mutations in the gene encoding SLURP-1 in Mal de Meleda. Hum Mol Genet 2001; 10:875-80.

107 Vanderhooft SL, Francis JS, Holbrook KA et al. Familial pityriasis rubra pilaris. Arch Dermatol 1995; 131:448-53.

108 Nagy N, Wedgeworth E, Hamada T et al. Schopf-Schulz-Passarge syndrome resulting from a homozygous nonsense mutation in WNT10A. J Dermatol Sci 2010; 58:220-2.

109 Adaimy L, Chouery E, Megarbane H et al. Mutation in WNT10A is associated with an autosomal recessive ectodermal dysplasia: the odonto-onycho-dermal dysplasia. Am J Hum Genet 2007; 81:821-8.

110 Blaydon DC, Etheridge SL, Risk JM et al. RHBDF2 mutations are associated with tylosis, a familial esophageal cancer syndrome. Am J Hum Genet 2012; 90:340-6.

111 Toomes C, James J, Wood AJ et al. Loss-of-function mutations in the cathepsin $\mathrm{C}$ gene result in periodontal disease and palmoplantar keratosis. Nat Genet 1999; 23:421-4.

\section{Supporting Information}

Additional Supporting Information may be found in the online version of this article at the publisher's website:

Video S1 Author video. 